\title{
Spatial Dimensional Shift in Graphic Design and Visual Communication
}

\author{
Jiang-Hui Liu* \\ Education Technology Center \\ Guangdong University of Foreign Studies \\ Guangzhou, China \\ 247031690@qq.com
}

\author{
Han-Dun Xiang \\ College of Art \\ Guangdong University of Foreign Studies \\ Guangzhou, China
}

\begin{abstract}
In the process of decoration and graphic design, it is often necessary to reflect a three-dimensional image. How to better transform two-dimensional graphics into threedimensional rendering in the two-dimensional state of the plane is what designers need to think and learn. Visual illusion is a kind of antagonistic form in which one's visual sense organs are stimulated by external environment and contradict the real psychological reaction. In this paper, the theory of visual illusion and the complexity, comprehensiveness and crossover of visual communication design are used to discuss the three-dimensional transformation under various factors. The results show that with the development of the times, the ingenious use of visual illusion in the essential two-dimensional state of plane can change dull two-dimensional space to the visual sense of the newer threedimensional space, so that viewers can achieve a new visual enjoyment and rich visual expression.
\end{abstract}

Keywords-Visual illusion; Plane design; Three-dimensional space; Visual Communication

\section{INTRODUCTION}

In daily life, there are many external factors, such as colour, light and graphics, that may cause our visual and psychological ambivalence. Under the condition of the natural human had little chance to appear on the vision because of personal subjective psychological and sensory accept foreign is not the same as the real response. This will make the audience to think, things, the two-dimensional in the form of ordinary life has widespread. When properly purposefully to join some external environment change, can make it in two-dimensional form skillfully use all kinds of external environment and achieve the three-dimensional visual illusion [1]. These external visual illusion can guide their inner thinking and exploration, Will not only make the audience feel refreshed. This form will also promote the development of two-dimensional design in the field of graphic design and innovation. For the plane design field elements including the material, graphics, colour, text, compared with the traditional thinking, these are all in a twodimensional fixed thinking mode of the breakthrough point, which is the same. In the people's thought developing environment, obviously can't provide visual enjoyment and spread very well.

\section{THE CONNOTATION AND PRINCIPLE OF VISUAL ILLUSION}

In the broad sense of illusion, touch, hearing, vision, etc. A phenomenon produced by the organs of the senses which are used to sense the world. Visual illusion is a very important way of experiencing the world, $\mathrm{He}$ accounts for 85 percent of the world we live in. As the focus of this article. Visual illusion is produced under certain environmental impact of a Marxist dialectical materialism of the objective conditions of an act of bending the truth, for the traditional concept. The ear is virtual, seeing is believing. Give new negatives. Here the visual illusion brings to people a new subversion of the inconsistency of the visual perception. [2] From the perspective of external factors, visual illusion is affected by many aspects and multiple levels, such as lines, colours, rehearsal methods, shapes, etc. $\mathrm{He}$ also included within the brain response in the form of a series of many complex. Control vision convey occipital lobe of the brain cortex and closely combined with the role in visual orientation of brain nerve system. When people use eyes to observe a particular object, the object through the light reflected light reflection to enter the pupil and the lens. After the light penetration, scattered on the retina. The retina are attached to the decent through photoreceptor cells and light signals generated by using light into chemical signals through a variety of combined neural responses from eventually accept standing within the cerebral cortex to the brain. The brain through the analysis, cognitive,

Coming from a memory to the outside world The colour, size, shape and other concepts of the series are judged and expressed in a unified way, so that the vision is generated. From the above, the visual is not our traditional cognitive vision. He is through mental and visual sense and a series of complex forms of synthesis. Explore the visual illusion, that is, on one of the nodes under the influence of these integrated pattern of behavior that an error has occurred and the error of the node is not subjective control [3]. He is a passive behavior, everything is born to accept specific regularity summary of three dimensional things. In the daily life of all stripes, need to avoid the formation of the illusion. But will he applied to design fields, He will bring new innovation to current design. Can greatly promote the development of design field, enhance the impact of the visual performance. To guide people's visual and psychological contradictions, make the audience from the previous passive behavior patterns, to the present automatic 
appreciation and thinking process. So as to promote the designer and the audience interaction, enhance product interesting and interactive.

\section{THE INNER ELEMENTS EXPLORE THE THREE- DIMENSIONAL FORM}

\section{A. Three dimensional transitions in colour}

Colour produced in various basic primary colours mixed colours and colour contrast in space, a variety of different colour combinations. Even the same colour also can appear many different combinations. For control has three elements of colour, hue, lightness and saturation. These colours can be infinitely separated a new colour, but in so many colours. Is this colorful world, we can imagine the world without colour will how dull. So in the category of visual colour, visual illusion is also plays a very important role. The colour itself is not weight. But the human emotion can be artificially through other ways for him and have a lot of weight to our design. In the form of different colour also gives a new performance by us, this is due to the psychological characteristics and the results of all. When you see a colour, we will have different psychological feeling. When he saw the red, he can bring us hope, a warm, positive, outbreak, when see the combination of blue and white colours. Will feel cool, so cool, when he saw the green, he brings us psychological feeling of vitality. On the other hand. Fill in different colours of the same shape, give a person the size of the feeling is not the same. Of course, can bring a three-dimensional sense is that colour can bring deep sense, this is the main form of three-dimensional.

In the Renaissance era, painting has entered a new era. The painter has broken the traditional painting with sensing, and developed many scientific theories. On colour, the original images and no sense of depth. One problem is basic it is to use a colour picture. Until 1666, when the great physicist Newton's prism to decompose the natural light. It is concluded that the light is made up of seven colours. Therefore, the scientific painting of nature finally developed rapidly at this time. In these practices, painters came to the conclusion that warm colours can bring us the feeling of standing out from the front, while cool colours can bring us the feeling of stepping back A famous brutalist painter Henri Matisse's (1869-1954) Lady mattis of green stripes, in Figure 1. Artist seems to be mean to change the colour of the picture on the left to the high saturation of warm colour. On the right side of a small piece of the complete place and appears as a high degree of saturation of green. Make people feel chunks of warm colour is a flat area, green is an area. But the picture of the green and red produces a visual illusion formed a deep distance, two colours in the picture is a set of contrasting colours. The picture of tonal very prominent had magical powers. The painter painting such an ordinary, The effect is the active result of mattis' thought. It can be seen that his extraordinary painting skills, rigorous thinking and his ability to control colour depth.

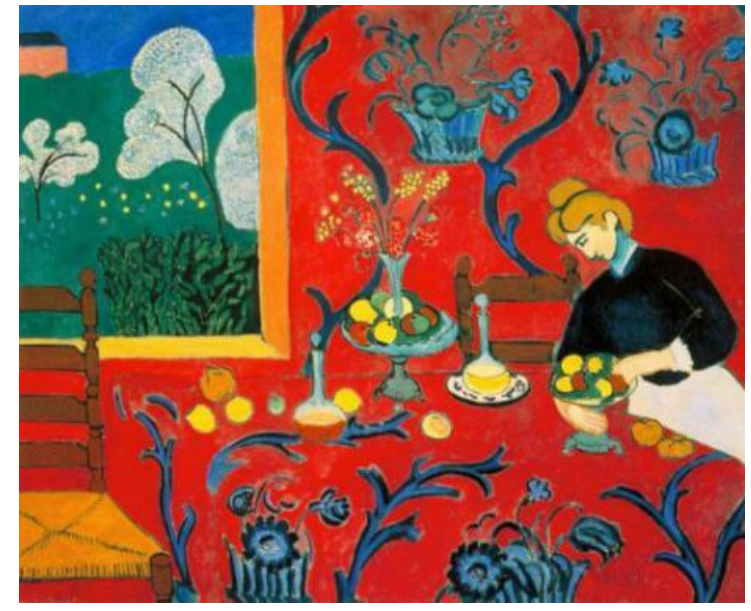

Fig. 1. Lady mattis of green stripes.

\section{B. Three-dimensional transitions in graphics}

Graphic design has a long history, the earliest can be traced back to Rome in Edinburgh first newspaper published. At that time in the newspaper has a similar pattern is used to explain the text form of illustration, from the modern plane design is based on the German Bauhaus school leadership of modernism. One of the representative style is abstract, but no matter how to change these forms. People are in the thinking formulary of the two-dimensional plane. Think that the plane design as its name implies is the form of plane. From Bauhaus to the present, the course of planar composition has been the focus of the teaching of visual communication design in modern universities.

To create new forms of attitude is important in the field of design thought. And the concept of three dimensional space will be a very good innovation. In the design of visual illusion, there are many illusion, such as the length of the line illusion as shown Figure 2 Muller Lyle found in 1889. Mueller Lyle illusion of two equal length line alignment is put up and down, on both ends of a line segment and extending outward in another line both sides of the bracket and extends inward brackets. The vision of people would have a different behavior and objective facts. Think of two line a is longer than the other.

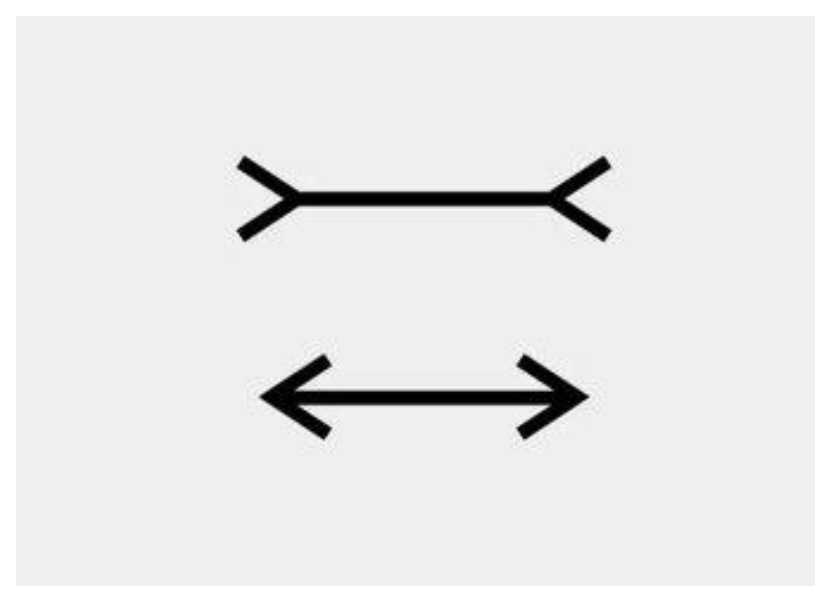

Fig. 2. Mueller Lyle illusion. 
There is also an illusion in the area of the figure, and there are many uncertainties related to the influence of area [3]. Different angles of the same object, different directions of the same object, different brightness of the same object, etc., will cause different size changes of the object visually As shown in Figure 3. One is a black circle on a white background. The other is a white circle on a black background. Our vision then deceives us that white vases are larger than black vases.

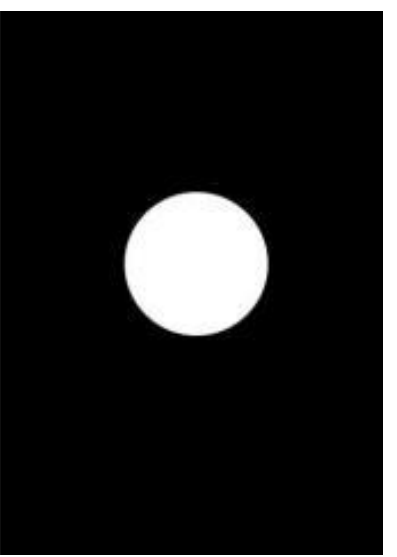

Fig. 3. Circles on different backgrounds.

Of course, these illusions in two dimensions are not important content. The most important core of the transformation from two dimensions to three dimensions is the following spatial illusion. Specifically, it is the contradictory space, which does not exist in real life. If not carefully to taste, contradiction space may also won't feel strange. As long as seriously to comb the entire story, will find that actually happened in visual effect and objective contradictions. In twodimensional plane to simulate realistic contradiction space, create a kind of philosophical effect and unique space to experience. Can bring to a very strong vision wallop, stronger increased interest. It can make many levels of contradiction effect and the spatial relationship. Give a person a kind of unexpected strong visual perception of space. Thus contradiction space has several important features. Contradictory, planar and hallucinatory characteristics [4].Producing a good spatial effect can enhance the expression of the design. When we talk about the contradictory space, we have to talk about the founder of the contradictory space. Works of the masters are used to the extreme example. Seemingly are reasonable, but as long as a careful analysis would be found in the form of these works which is not objective, by using the method of contradiction space to create mutual dislocation overlapping contradiction space. At the same time, the viewer can be attracted to these impact. Realize the meaning of these works.

In logic and linguistics, an idea is "self-reference" or "refers to" say a sentence to describe this sentence itself, such as the "nine words are there in this quote," it's not English ", "when you read these three examples are already feeling the strong interest". This cycle, recursion and iteration statements can often create intriguing realm, we can in Escher's work experience this rare genius is how to use the space created a fascinating work. Other self-referential work shows him more outstanding talents, Escher's "psycho-game-like visual illusions" have a similar ring to them.

Since the paradoxical nature of Escher's works determines that reality is unsolvable, how to restore the "truth"? In fact, existence is reasonable, the claim itself exists paradox, but still "the truth", because the creation of the "truth" is the "illusion". Escher's famous work, Viewing Tower, as shown in Figure 4, is clever use of Jelinek Square principle, different connection point is using the cube to create the cube "impossible". The structure of the cube is divided into different regions, and the layers of these connection points create the existence of contradictions under different Spaces, so that people have the contradiction of "seeing is real", which is actually unreasonable "seeing is wrong" psychology, as shown in Figure 4.

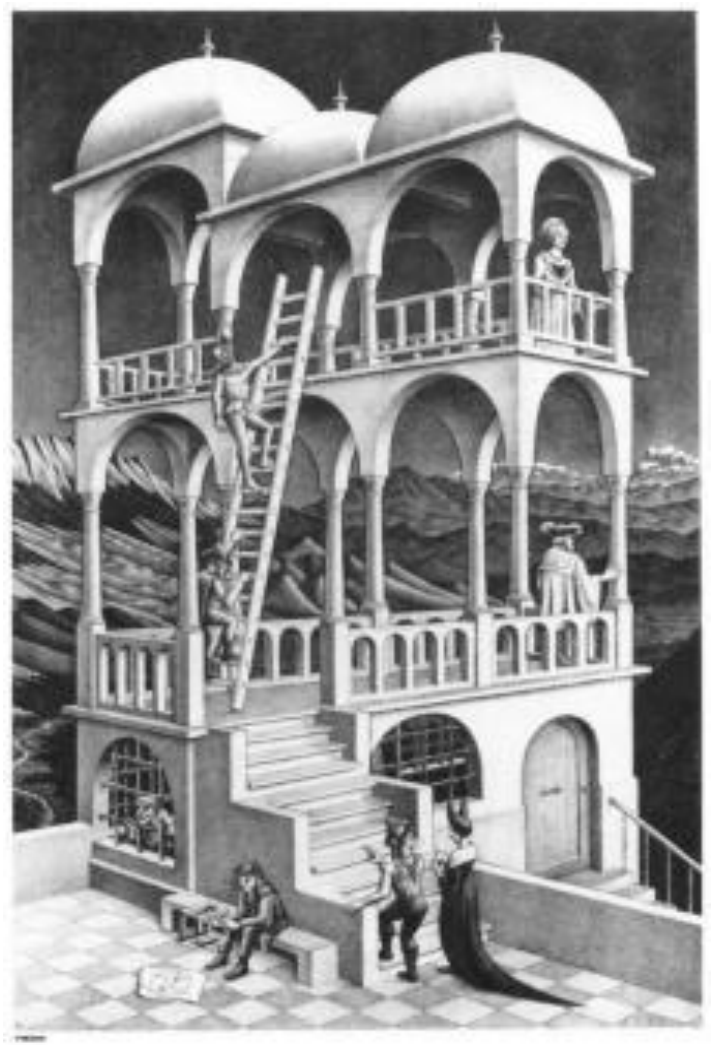

Fig. 4. Viewing Tower by Escher.

\section{Three dimensional transitions in text}

Text from the perspective of a specific case, in fact, as early as the oldest words produced three-dimensional characters. Although at that time the three-dimensional performance is not conscious, but now still offer a three-dimensional reference form. Then the three-dimensional text mainly includes the Yin line lettering and Yang line lettering, one is the surface with sharp objects carved concave marks, another is the relief form. In these forms have the font of space and volume. In modern times, with the development of science and technology, people have preliminarily explored the three-dimensional font space, for example, the stereoscopic font in the form of projection is adopted in the printing poster by laser printing fonts. People 
gradually broke through the text two-dimensional pattern. The font design in every design industry has a very important role.

The passive anaglyph three-dimensional his evolution from the ancient to the 20 to 60 s of the 20th century the plane of the typeface design and the initiative to break on a two dimensional form to the modern three-dimensional development of the $21 \mathrm{st}$ century. In the form of threedimensional font has a natural three-dimensional is a kind of form. Because the person is three-dimensional and the burden to the concept of mental or emotional, can let a plane to express three-dimensional fonts appear very real to touch. Appreciate the people great pleasure. In modern times with the improvement and development of printing technology, there will be different forms of new fonts. Such as the lettering will appear different on different new material of three dimensional feeling, change the way plane font to the three dimensional form mainly through the perspective rule to express the font three-dimensional performance. On the same object to the use of different methods of perspective will bring different threedimensional effect. Make the font more rich expression and different levels of stereo. There are several kinds of commonly used form of perspective,

The most common is a little perspective, that is, linear perspective. Such as bridge see the bridge on both sides of the line has been extended to the most distant and collection for the vanishing point. Before and after object overlap effect also can produce three-dimensional form of illusion, have into the object from us far away from us small. And the camera nearly far the concept of virtual reality, visual illusion, as a kind of means in the form of font can be more rich. Greatly enhanced planar space sense of reality. As shown in Figure 5, Japanese art talent Nagai Hideyuki's paper three-dimensional stereoscopic sketching presents amazing visual creativity. The work is an amazing three-dimensional visual feast with the illusion of perspective.

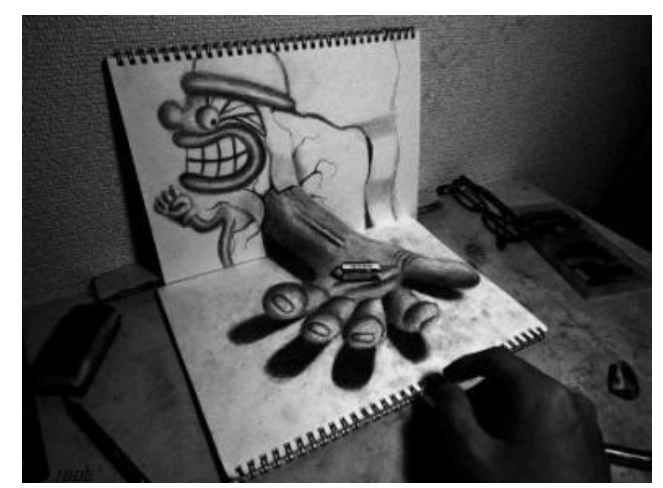

Fig. 5. Nagai Hideyuki's paper three-dimensional stereoscopic sketching.

The second point is to use the combination of rehearsal and density of words to regard the font as the form of point and line, so that the text in the static form produces the threedimensional sense of movement in the plane. It will create a sense of flow, giving people a strong overall three-dimensional sense of backward [5]. Thirdly, the effect of overlapping words creates a sense of heaviness that our vision mistakenly assumes is weighted and blocky. The surface of the text and the surface of the text are combined in different ways at different angles [6]. Through the combination, a certain three-dimensional inner space illusion in two-dimensional plane is generated, as shown in Figure 6.

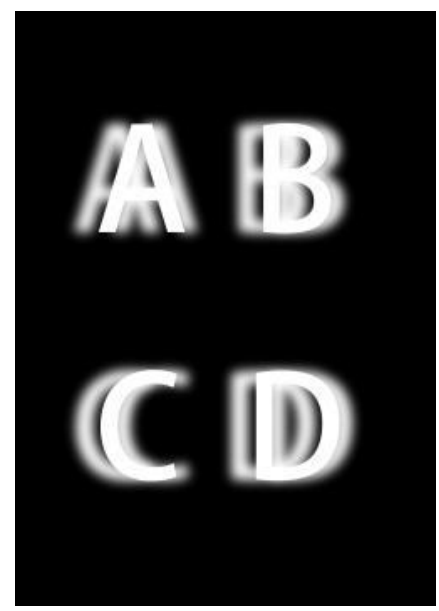

Fig. 6. Three-dimensional inner space illusion in two-dimensional plane.

\section{CONCLUSION}

Come along with the continuous development of science and technology in the 21 st century, the viewer's eyes constantly improve. Visual illusion in the field of design will lead twodimensional comprehensive into a three-dimensional form. Even break through the boundaries of design, to develop in the direction of more generalized. Makes stereo vision are common in all things, to become a one integrated mass of three-dimensional family. Maybe in the near future the threedimensional form of expression will be replaced by the more colorful the four-dimensional form, four dimensions and then replaced by five dimensions. In this, the manifestation of visual illusion will be the most critical innovation drive, guiding the development of society.

\section{REFERENCES}

[1] Y. X. Xiao, "Application of three-dimensional shape in graphic design," in Packaging Engineering, 2018. In Chinese.

[2] Y. R. Niu, Application and Research of Visual Illusion in Graphic Design. Shanxi, Shanxi Normal University, 2015. In Chinese.

[3] W. J. Zhao, Effect of Color on the Transverse Parallel Illusion of Line Segment and Eye Movement Study. Zhejiang, Zhejiang University of Science and Technology, 2015. In Chinese.

[4] D. M. Du, "The ambivalence space in advertising graphic design, " In Anhui Literature, vol. 6, pp. 198-199, June 2011. In Chinese.

[5] H. Dai, A Study on the Stereoscopic Visual Representation of Fonts. Nanjing, Nanjing Art Institute, 2016. In Chinese.

[6] H. L. Wei, "Application of three-dimensional form in graphic design," in Art Science and Technology, vol. 26, pp. 193, 2013. In Chinese. 